\title{
The next 25 years
}

\author{
R B GOUDIE
}

From the University Department of Pathology, Royal Infirmary, Glasgow, Scotland

Since its foundation 25 years ago the Royal College of Pathologists has played a major part in the development of pathology in this country. Its advice to the Department of Health and Social Security on the level of staffing of district general and teaching hospital laboratories and on the need for specialists in histopathology, microbiology, pathological biochemistry and haematology has had an important influence on the direction and extent of the expansion that has taken place in the laboratory services. In addition, the insistence by the College on adequate training of pathologists in recognised laboratories and the establishment of a demanding final examination, which is virtually essential for appointment to consultant or senior scientific posts in National Health Service pathology departments, has greatly improved the quality of the service provided to patients.

Pathology itself has also changed. Molecular biology has arrived on the scene; automation has taken over more and more of the tasks previously performed by people; increased output of doctors by the universities has improved medical recruitment to the laboratory specialties; and needs for additional laboratory tests have arisen because of changes in medical practice, including larger numbers of consultants in the subspecialties of medicine and surgery, and the use of new imaging techniques and methods of treatment. Furthermore, from never having had it so good in the 1960's, our teaching hospital laboratories are now suffering from cuts in university and Medical Research Council budgets and reallocation of NHS funds from hospital to community and from centres of excellence to periphery. What, then, is going to happen to laboratory medicine in the next 25 years, and what can the College do to foster the subject?

\section{Scientific advances}

Pathology is the science of disease, and new scientific knowledge will profoundly affect the way in which it is practised in the future. By far the most important developments in all branches of the subject will almost certainly stem from the new found ability of the molecular biologists to identify and isolate individual genes and polypeptides and produce them in large amounts in vitro.

Investigation of the molecular basis of microbial pathogenicity will probably have the most immediate and obvious impact on human welfare by leading to the discovery of new antigens for prophylactic immunisation. Recombinant DNA technology will then permit large scale production of antigen molecules or the creation of recombinant hybrid organisms for use as live vaccines. Hopefully, these will help to control major public health problems, varying from the common cold to malaria and the acquired immune deficiency syndrome (AIDS), though success will be limited by the evolution of vaccine resistant variants among pathogenic organisms. Molecular biology is likely to have other important effects on medical microbiology. New therapeutic agents will be developed as further molecular differences are discovered between micro-organisms and their hosts. Study of diseased tissues with probes for microbial genes and gene products will indicate infective causes for a number of neoplasms and chronic inflammatory and degenerative disorders. These may include some of the more common diseases of arteries, nervous system, and joints, and open the way for important preventive measures. The use of molecular finger printing in the taxonomy and typing of micro-organisms will provide a powerful new tool in microbiological diagnosis and in the epidemiology of infectious disease.

The new DNA technology has already led to great advances in clinical genetics. Within a few years the genes responsible for all the more common serious inherited disorders will be fully characterised and reliable methods developed for genetic counselling and the diagnosis of affected fetuses, if termination of pregnancy is to be considered. Success in treatment of some innate enzyme deficiencies by transplantation of normal bone marrow has already been reported, and such disorders may in future be corrected by transfection of the patient's own cells with normal genes. As more genes are characterised, opportunities will arise to understand the more complex polygenic diseases in which more than one locus participates, and to provide a scientific basis for investigation of the pathogenesis and epidemiology of the many disorders in which both heredity and environment play an 
important part.

In the longer term, there are some 50000 genes to be characterised in the human genome, and much research will be directed to exploring how the expression of each gene is controlled and the function of its polypeptide product. The effects of the simultaneous and sequential activation of groups of genes will also need to be considered. Most of this research will be done by workers in the basic biological sciences, using cell cultures and experimental animals, and a huge new physiology will gradually emerge. Pathologists and clinicians will have important roles in applying these developments to medical practice. First, they will help to identify possible applications of the new knowledge and provide the basic scientists with pathological specimens from appropriately chosen, well documented patients. This will best be done by those with special clinical interests and understanding of the assessment of laboratory tests. Clinical biochemists will also set up assays in their own laboratories to measure newly discovered molecular markers in body fluids and in tissue homogenates, while histopathologists and cytopathologists will investigate their occurrence and localisation in pathological tissues using immunocytochemistry and in situ nucleic acid hybridisation. Last, and perhaps most importantly, pathologists with a first hand knowledge of human disease and of molecular biology may make substantial contributions to the clinical problems they encounter in their daily work, such as the nature of the molecular changes in neoplastic cells that permit continuous replication and growth and metastasis, information which would be of great value in the diagnosis and management of tumours in man.

We can thus predict the introduction in the next 25 years of hundreds of new molecular biology laboratory tests which will be of immediate importance in patient management or preventive medicine. Many more will be devised that will initially be of little or no practical value, though some of these will eventually point the way to a better understanding of disease and the development of new forms of treatment.

\section{Technical advances}

Future progress in chemistry and the design of equipment will ensure the further development of methods to automate, miniaturise, simplify and speed up all forms of pathological investigation.

Nucleic acid hybridisation, using inexpensive sandwich techniques and non-radioactive labels, will revolutionise current microbiological practice, permitting detection of infective agents directly from clinical samples in side-room or laboratory, and thus making the investigation of infection an immediate diagnostic service rather than a confirmatory one. Immuno- assays and new applications of physical measurements, such as the circular intensity differential scattering spectrum and Fourier transformation infrared spectroscopy will also contribute to the rapid identification of bacteria.

Whole blood analysers capable of performing most routine biochemical and immunoassays will be developed for operation by nurses and junior clinical staff. Calibration, quality control, and recognition of user error will be built in to the equipment and dilution of specimens and pipetting will be unnecessary. Coded access to the equipment will restrict its use to those who have received requisite training. The cost efficiency of completing tests within a few minutes of obtaining the specimen will make the use of such methods for near patient testing in clinics and ward side-rooms essential, especially when results are urgently required for therapeutic monitoring and in emergencies when rapid diagnosis may be life saving.

Continuous biochemical monitoring of the patient by miniaturised electrodes and other biosensors will be commonplace in intensive care units. Magnetic resonance and infrared spectroscopy will allow biochemical imaging of focal disease in vivo and will have an important place in the diagnosis of inflammatory and neoplastic lesions and maybe even in the investigation of psychiatric disorders.

\section{Laboratory finance}

One prediction can be made with certainty. The explosion of medical science and technology and the increasing number of consultants with special clinical interests will make subspecialisation in pathology and increased spending on materials and staff necessary for the provision of laboratory services.

In the next 25 years much more attention will therefore be given by government and health service administrators to getting the best value for money from diagnostic laboratories, and two methods are likely to be attempted.

The first will be the use of performance indicators relating the amount of work done by individual laboratories to the overall cost. Measurement of amount of work done will inevitably be crude (it may not even take the difficulty or complexity of different kinds of test into consideration), while estimates of cost will probably exclude such factors as the price for patient or health service of delays in laboratory reporting. More importantly, performance indicators will take no account of the quality of work done or whether the tests performed are relevant to patient management or clinical research. Extreme difficulty will be encountered in making rational policy decisions from data on performance indicators.

The second method will aim simply to contain costs 
by giving medical practitioners annual laboratory test budgets which they must not exceed, the assumption being that the clinician is best able to decide priorities in the use of expensive laboratory resources. Clinical budgeting will inevitably have an adverse effect on laboratory performance indicators due to fluctuating demand for tests and reduced laboratory cash flow, as clinical budgets become exhausted towards the end of the financial year. More importantly, clinical budget holders may, in the interests of economy, use their budgets to purchase near patient analysers for their own needs, or make use of cheap privately run laboratories, so reducing the funds available for departments that provide general laboratory services and training for personnel etc.

\section{Laboratory management}

The present and by far the best administrative arrangement is that in which appropriately trained laboratory consultants or, in some disciplines, science graduates of equivalent status, are budget holders in overall charge of hospital laboratories. This will be challenged by physicians and surgeons who would prefer to run specialised laboratories for their own particular needs; trades unions and other organisations representing medical laboratory scientific officers who see themselves as the laboratory managers of the future; and health service administrators, whose job it is to try to find alternative mangement schemes that will lead to cheaper pathological services.

Haematology as it is organised now-a laboratory discipline providing biochemical, serological, cellular and morphological investigations for patients with a restricted group of diseases by a consultant who acts both as clinician in charge of beds and pathologist in charge of the laboratory-may be a model of the shape of things to come in other subspecialties, such as gastroenterology, gynaecology, endocrinology and dermatology. This would overcome some of the difficulties of pathologists who run general histopathology or biochemistry laboratories in keeping up with the complex and changing problems of the many subspecialties of medicine and surgery they serve. It will, however, become increasingly difficult for individuals to achieve and maintain proficiency in both clinical and laboratory practice, and if this is widely attempted, there is the danger that while they take care of their clinical commitments the management of the laboratories will slip into the hands of others. A fragmented, purely technical laboratory service will then evolve, lacking in leadership, with a sound knowledge of the biological and clinical aspects of laboratory practice but incapable of providing a balanced service to meet the laboratory requirements of the local community.

\section{Other developments}

The pattern of disease in the community will continue to change, and AIDS will remain a major preoccupation. Because the human immunodeficiency virus (HIV) genome is integrated in host cell DNA, it is unlikely that there will be any form of treatment capable of completely eliminating the infection, though drugs have been found which, while they are being administered, improve the clinical condition of sufferers and render them non-infective to others. An effective AIDS vaccine may be developed, though it may not be used in time to prevent the disease in members of the community exposed to infection by their sexual activities.

Other hitherto unknown infections will come into prominence, some as a result of being discovered by the application of new diagnostic techniques, some due to social and environmental changes in human populations, and some because of genetic changes in the micro-organisms themselves.

Molecular probes for the study of the homeobox and other genes associated with developmental abnormalities will revolutionise the diagnostic classification and prevention of dysmorphic syndromes and some forms of mental subnormality.

Large numbers of "new" metabolic disorders, inherited or acquired from drugs or other environmental factors, will be characterised, and computerised information systems as well as special laboratory tests will be required to facilitate their diagnosis.

New drugs acting on growth factors or their receptors will be used in the treatment of cancer, but their beneficial effects are likely to be of limited duration unless progress is made in developing methods to prevent the emergence of drug resistant tumour cell lines. Improved histopathological classification of tumours will lead to the recognition of new subtypes and the discovery of new environmental carcinogens. With adequately trained cytopathologists and improved sampling methods, rapid examination of cells obtained from mucosal surfaces by endoscopy or from solid lesions by fine needle aspiration will largely replace conventional biopsy techniques in preoperative surgical diagnosis.

Despite their growing clinical importance, molecular biology and immunology are unlikely to achieve the status of major independent disciplines in hospital pathology because of their overlap with the older established laboratory specialties, though regional laboratories providing services in molecular genetics and cytogenetics will expand to provide support for clinical geneticists, obstetricians, and pediatricians. Similarly, regional immunopathology laboratories, 
often associated with academic departments of immunology, will supplement the immunological services provided by hospital laboratories in the other disciplines and give much needed guidance on basic aspects of the subject.

\section{Future role of the College}

In the years ahead the College must give a lead to government and the professions in the development of laboratory medicine. The predictions outlined above are based on present trends. Most of the important problems to be encountered will come from the continuing expansion of science and technology.

Existing laboratory resources are already inadequate, and the College must press government for additional funding for more pathological research in the universities and for the exploitation of new tests in NHS laboratories. It must be made clear, hopefully with the support of the other Royal Colleges, that increased numbers of consultants in medicine and surgery require at least a corresponding increase in pathologists and that the introduction of new health programmes, such as mass population screening for cancer, demands additional laboratory resources.

The need for appropriately trained senior medical staff with a commitment to laboratory work must be emphasised. Their importance in laboratory management has already been discussed. They also have an essential role in keeping abreast of current devel- opments in the discipline, in updating laboratoryo practice, in advising and educating clinicians in the use and limitations of laboratory tests and in the interpretation of laboratory results. There are press ing needs for subspecialisation with each laboratory discipline to cope with the increasingly diverse ande detailed laboratory requirements of the growing num $\frac{\bar{D}}{\bar{D}}$ ber of subspecialists in clinical medicine and surgery $\frac{\text { के }}{\sigma}$ The College should have firm advice to give on this subject, especially on the relative roles of regional ref $\frac{\text { es }}{2}$ erence laboratories and district hospital laboratoriesand on the manpower required.

Last and by no means least, the present somewhat $\vec{\epsilon}$ general College final examination in each of the maine laboratory disciplines, well tried and successful as if has been, is failing to provide suitable applicants for consultant posts for which work experience iso required in a subspecialty such as cytopathology. The problem of specialist training is, of course, related to $\vec{r}$ uncertainties about future specialist staffing levels ando organisation of laboratory services at district,, regional, and national levels. The evolution of pathol- $\bar{c}$ ogy in the next 25 years will depend largely on itso ability to adapt to change. The College must adjust its training and examination requirements to meet the 3 demands of continuing rapid advances in the basic and clinical sciences.

Requests for reprints to: Professor RB Goudie, Departme of Pathology, Royal Infirmary, Glasgow G4 OSF. 